\title{
Time-Related Changes in Detrusor Overactivity in Awake Rats with Spinal Cord Injury Observed by Simultaneous Registrations of Intravesical and Intraabdominal Pressures
}

\author{
Long-Hu Jin, Hwa-Yoan Shin, Seung-Hwan Yoon ${ }^{1}$, Do-Hwan Seong, Chang-Shin Park², Tack Lee, Sang-Min Yoon \\ Departments of Urology, ${ }^{1}$ Neurosurgey, and ${ }^{2}$ Pharmacology, Inha University School of Medicine by BK21 project, Incheon, Korea
}

\begin{abstract}
Purpose: To compare the physical characteristics of detrusor overactivity (DO) induced by intravesical infusion of saline in awake, sham rats and rats with chronic spinal cord injury (SCI), by simultaneous registrations of intravesical and intraabdominal pressures.

Methods: Male Sprague-Dawley rats, normal or with a spinal vascular clip at the level of Th9, were investigated cystometrically 1 and 4 weeks after SCI. Intravesical pressure (IVP) and intraabdominal pressure (IAP) were recorded simultaneously to evaluate true DO. During the filling phase, the event of IVP rises, defined as increments that exceeded $2 \mathrm{cmH}_{2} \mathrm{O}$ from baseline, were determined as DO according to the absence of simultaneous changes in IAP.

Results: All SCI rats exhibited DO during the filling phase, which was not shown in sham rats. The frequency and pressure of DO had a tendency to decrease with time. The DO frequency of SCI rats after 4 weeks $\left(0.9 \pm 0.2 \mathrm{~min}^{-1}\right)$ was decreased compared with that after 1 week $\left(2.1 \pm 0.4 \mathrm{~min}^{-1} ; \mathrm{P}<0.05\right)$. The DO pressure of SCI rats after 4 weeks $\left(8.4 \pm 1.9 \mathrm{cmH}_{2} \mathrm{O}\right)$ was decreased compared with that after 1 week $\left(11.6 \pm 2.9 \mathrm{cmH}_{2} \mathrm{O} ; \mathrm{P}>0.05\right)$.

Conclusions: Cystometric studies in awake male SCI rats showed some significant changes in bladder function after SCI. All SCI rats exhibited DO during the filling phase, and showed different physical characteristics of DO over the course of time. The neurological basis of these time-related changes remains poorly understood, but may provide important prognostic information about long-term urological management in SCI patients.
\end{abstract}

Keywords: Urodynamics; Overactive detrusor; Spinal cord injuries

\section{INTRODUCTION}

Injury to the spinal cord rostral to the lumbosacral level results in detrusor hyperreflexia, which presents as neurogenic detrusor overactivity (DO) urodynamically [1-3]. In humans and animals with paraplegia or tetraplegia, an initial variable period of urinary retention is followed by the appearance of hyperreflexic automatic voiding as a result of DO [4-6]. During the several years after the injury, micturitions are impaired owing to several causes, depending on the functional or anatomical bladder outlet obstruction (BOO) and/or changes in characteristics of the bladder itself as a result of this BOO [7-9]. However, no investigations have focused on the DO as the change in the bladder itself, which involvs the electrophysiologic changes in the reorganized and recovering spinal and supraspinal circuits during the time course.

In this research, we observed and compared the physical characteristics of DO induced by intravesical infusion of saline in awake, sham rats and rats with acute and chronic spinal cord injury (SCI) by simultaneous registrations of intravesical and
Corresponding author: Sang-Min Yoon

Department of Urology, Inha University Hospital, Inha University School of Medicine, Sinheung-dong 3-ga, Jung-gu, Incheon 400-711, Korea

Tel: +82-32-890-2360 / Fax: +82-32-890-3097 / E-mail: uroyoon@inha.ac.kr

Submitted: March 3, 2011 / Accepted after revision: March 20, 2011
This is an Open Access article distributed under the terms of the Creative Commons Attribution Non-Commercial License (http://creativecommons.org/licenses/by-nc/3.0/) which permits unrestricted non-commercial use, distribution, and reproduction in any medium, provided the original work is properly cited. 
intraabdominal pressures and compared voiding function in SCI and sham rats.

\section{MATERIALS AND METHODS}

\section{Animals}

Twenty-two age-matched (10-week-old) male Sprague-Dawley rats (Orient Bio Inc., Seongnam, Korea) weighing 205 and $255 \mathrm{~g}$ were used in this study. These rats were divided into four groups of sham and SCI rats that were evaluated at 1 (sham, 5 rats; SCI, 6 rats) and 4 (sham, 5 rats; SCI, 6 rats) weeks after the injury. All animals survived for the entire duration of the planned experiment, except one SCI rat in the 1-week group. All procedures were performed in accordance with the Guide for the Care and Use of Laboratory Animals of the National Institutes of Health and were approved by the local research ethics committee. The rats were maintained under standard laboratory conditions with a 12:12-hour light:dark cycle and free access to food pellets and tap water. Simultaneous intraabdominal pressure (IAP) and intravesical pressure (IVP) were continuously recorded during the filling and emptying phases of the cystometrogram, as described previously $[10,11]$.

\section{Surgical Procedures Spinal cord injury}

Rats were anesthetized by intraperitoneal (IP) injection of ketamine (75 mg/kg IP; Yuhan, Seoul, Korea;), and the laminectomy was done at T8-10 levels. A spinal cord injury was made by applying a vascular clip (Biemer-Clip, Aesculap, Tuttlingen, Germany) at the T9 level of the spinal cord for 15 minutes (occlusion pressure of $0.2-0.25 \mathrm{~N}$ ). The sham group underwent the same operation only without application of the vascular clip.

\section{Bladder and intra-abdominal catheter implantation}

Rats were anaesthetized with ketamine and xylazine $(15 \mathrm{mg} / \mathrm{kg}$ IP; Rompun, Bayer Korea, Seoul, Korea) 1 and 4 weeks after the injury to the spinal cord. After an abdominal incision was made, a polyethylene catheter (PE-50, Becton Dickinson, Parsipanny, NJ, USA) with a cuff was inserted into the dome of the bladder. To record IAP, a catheter with an abdominal balloon (Vinyl, Incheon, Korea) was inserted and attached with a silk tie to the posterior side of the bladder pressure recording catheter. The catheters were tunnelled subcutaneously and anchored to the skin of the back with a silk ligature. The free ends of the catheters were sealed. Each rat was housed individually after surgery, and food and water were given freely.

\section{Cystometric Investigations}

Cystometry was performed without anesthesia 3 days after the implantation of the bladder and abdominal catheters to obtain pressure- and volume-related urodynamic parameters. The conscious rats were placed in metabolic cages without restraint and the bladder catheters were connected via a T-tube to a pressure transducer (Research Grade Blood Pressure Transducer, Harvard Apparatus, Holliston, MA, USA) and a microinjection pump (PHD22/2000, Harvard Apparatus). Micturition volumes were recorded with a fluid collector connected to a forcedisplacement transducer (Research Grade Isometric Transducer, Harvard Apparatus). Room-temperature saline was infused into the bladder continuously at a rate of $10 \mathrm{~mL} / \mathrm{hr}$. Pressures and micturition volumes were recorded continuously with Acq Knowledge 3.8.1 software and an MP150 data acquisition system (Biopac Systems Inc., Goleta, CA, USA) at a sampling rate of $100 \mathrm{~Hz}$. The values from three reproducible micturition cycles were used for evaluation. IAP was defined as the recorded balloon pressure corrected by subtracting the lowest balloon pressure in each voiding cycle. The detrusor pressure (DP) was defined as the IVP minus the abdominal pressure. The DO during the filling phase was defined as increments of IVP that exceeded $2 \mathrm{cmH}_{2} \mathrm{O}$ from baseline and without simultaneous changes in IAP (Fig. 1).

The following urodynamic parameters were investigated: maximal pressure (MP, maximum bladder pressure during micturition), basal pressure (the lowest bladder pressure during filling), threshold pressure (TP, bladder pressure immediately before micturition), bladder capacity (BC, residual volume at the most recent previous micturition plus the volume of infused saline at micturition), micturition volume (MV, volume of expelled urine), residual volume (RV, bladder capacity minus micturition volume), micturition interval (MI), frequency of DO per minute, and increased amplitude of DO as DP (DO pressure).

\section{Statistical Analysis}

The results are given as mean values \pm standard errors of the mean. Normal distributions were confirmed by the ShapiroWilks' W test. Statistical significance was determined by Student's $t$ test and Bonferroni corrections as appropriate with statistical significance considered at $\mathrm{P}<0.05$. 

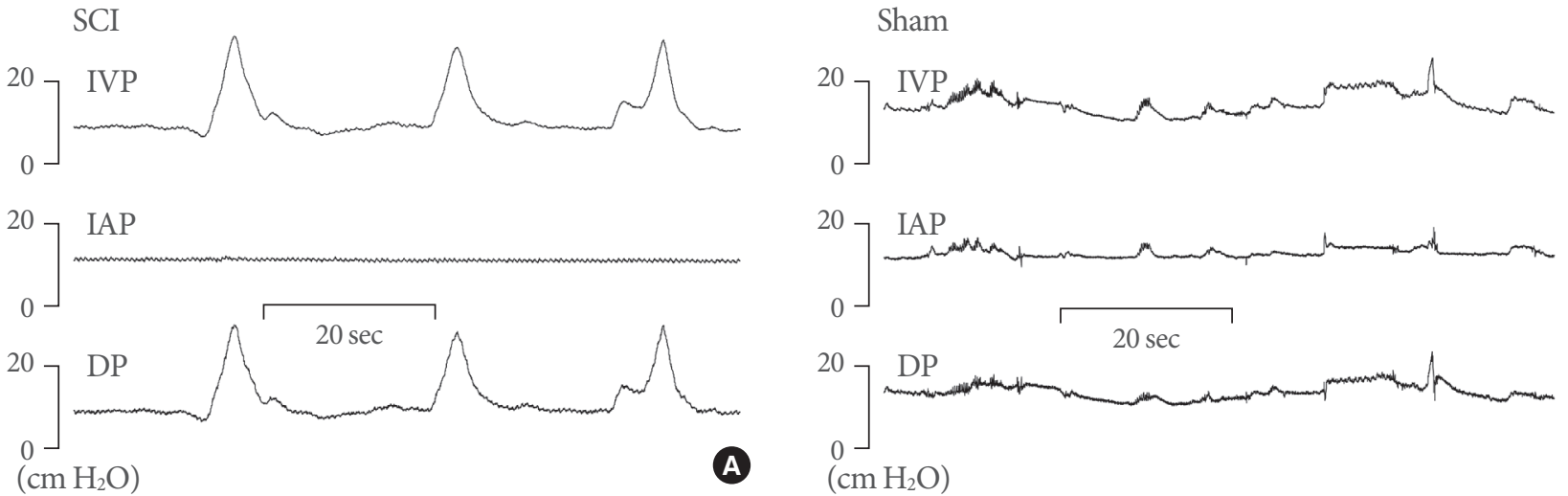

B

Fig. 1. Representative cystometrogram showing detrusor overactivity (DO) with intravesical pressure (IVP), detrusor (DP), and intra-abdominal (IAP) pressures in conscious spinal cord injury (SCI) rats (A), and sham rats (B). The IVP rises during the filling phase were interpreted as DO if occurring without simultaneous similar changes in IAP. DO was shown in SCI rats. Sham rats did not show DO but the abdominal straining.
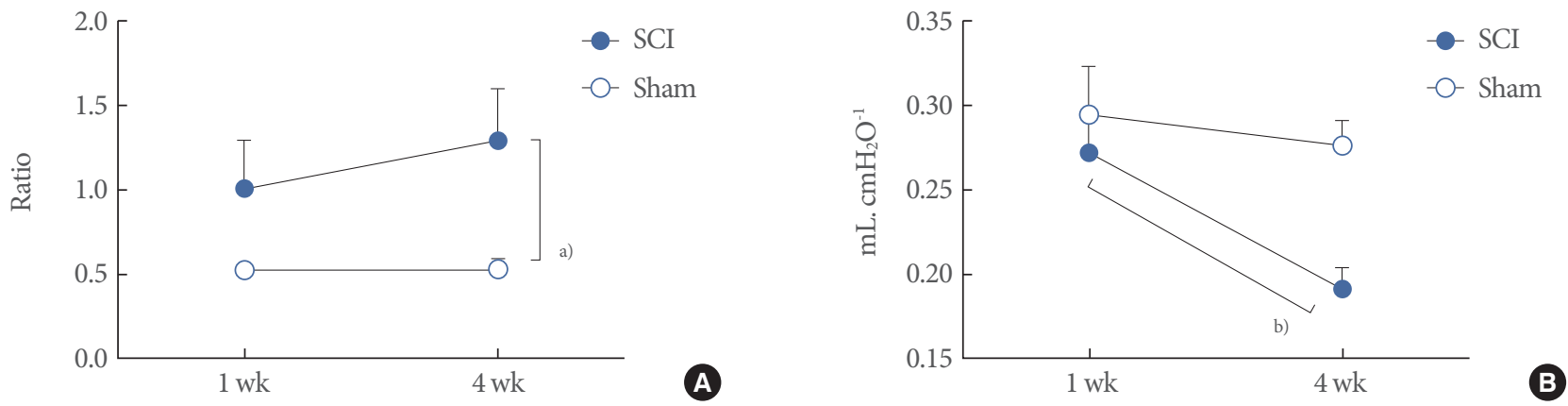

Fig. 2. The ratio of bladder weight (mg) to body weight (g) (A) and compliance (B) in spinal cord injury (SCI) and Sham rats. The ratio was higher in SCI rats than in sham rats at 4 weeks after the injury. The compliance of SCI rats after 4 weeks decreased significantly compared with that at 1 week. Results are expressed as the mean \pm standard error of the mean. ${ }^{\text {a) }} \mathrm{P}<0.05$. ${ }^{\text {b) }} \mathrm{P}<0.01$ (unpaired Student's t-test).

\section{RESULTS}

One week after the injury, the ratio of bladder weight (mg) to body weight ( $\mathrm{g}$ ) was not significantly different between the sham $(0.52 \pm 0.05)$ and SCI $(1.01 \pm 0.29)$ rats. However, the ratio was higher in SCI rats $(1.29 \pm 0.31)$ than in sham rats $(0.53 \pm 0.07) 4$ weeks after the injury $(\mathrm{P}<0.05)$ (Fig. 2A). The compliance of SCI rats after 4 weeks $\left(0.19 \pm 0.01 \mathrm{~mL} . \mathrm{cmH}_{2} \mathrm{O}^{-1}\right)$ decreased significantly compared with that at 1 week $(0.27 \pm 0.02$; $\mathrm{P}<0.01)$, which differed from the findings in the sham groups (Fig. 2B).

\section{Conventional Urodynamic Pressure- and Volume-related Parameters}

In the 21 female rats (10 sham and 11 SCI rats), 63 micturition cycles were assessed. In general, repeated cystometries provided reproducible results in both rats, but there were some differences in the pressure- and volume-related parameters of the cystometrograms between the groups (Figs. 3, 4).

Four weeks after the injury, SCI rats showed increased TP and MP by DP $\left(45.38 \pm 3.94,109.70 \pm 18.91 \mathrm{cmH}_{2} \mathrm{O}\right)$ compared with the age-matched sham rats $(24.18 \pm 2.68,60.05 \pm 6.057$ $\left.\mathrm{cmH}_{2} \mathrm{O} ; \mathrm{P}<0.05, \mathrm{P}<0.05\right)$, although there was no significant difference between them at 1 week after the injury (Fig. 3). BC was increased in SCI rats $(1.85 \pm 0.63 \mathrm{~mL})$ compared with sham rats 1 week after the injury $(1.12 \pm 0.13 \mathrm{~mL} ; \mathrm{P}<0.05)$. However, there was no significant difference between them 4 weeks after the injury, because of an increase in the sham rats' BC. As for $\mathrm{MV}$ and RV, there was no significant difference between the groups. However, SCI rats showed a tendency for increased RV over the time course without significance. MI inreased in SCI 

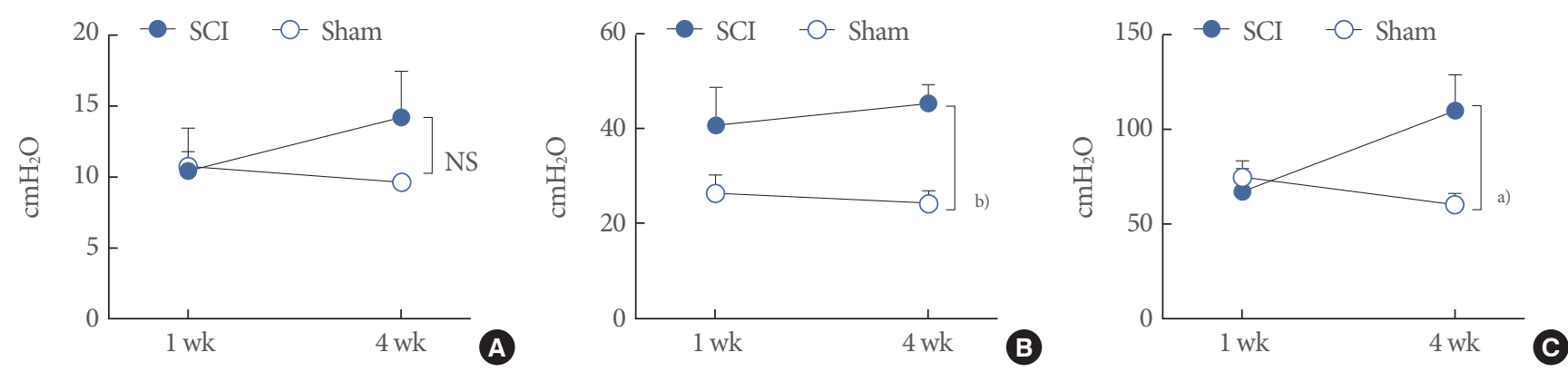

Fig. 3. The cystometric pressure-related parameters of awake spinal cord injury (SCI) and Sham rats. (A) Basal pressure by detrusor pressure (DP). (B) Threshold pressure by DP. (C) Maximum pressure by DP. Results are expressed as the mean \pm standard error of the mean. ${ }^{\text {a) }} \mathrm{P}<0.05$. ${ }^{\text {b) }} \mathrm{P}<0.01$ (unpaired Student's t-test). NS, no significance.
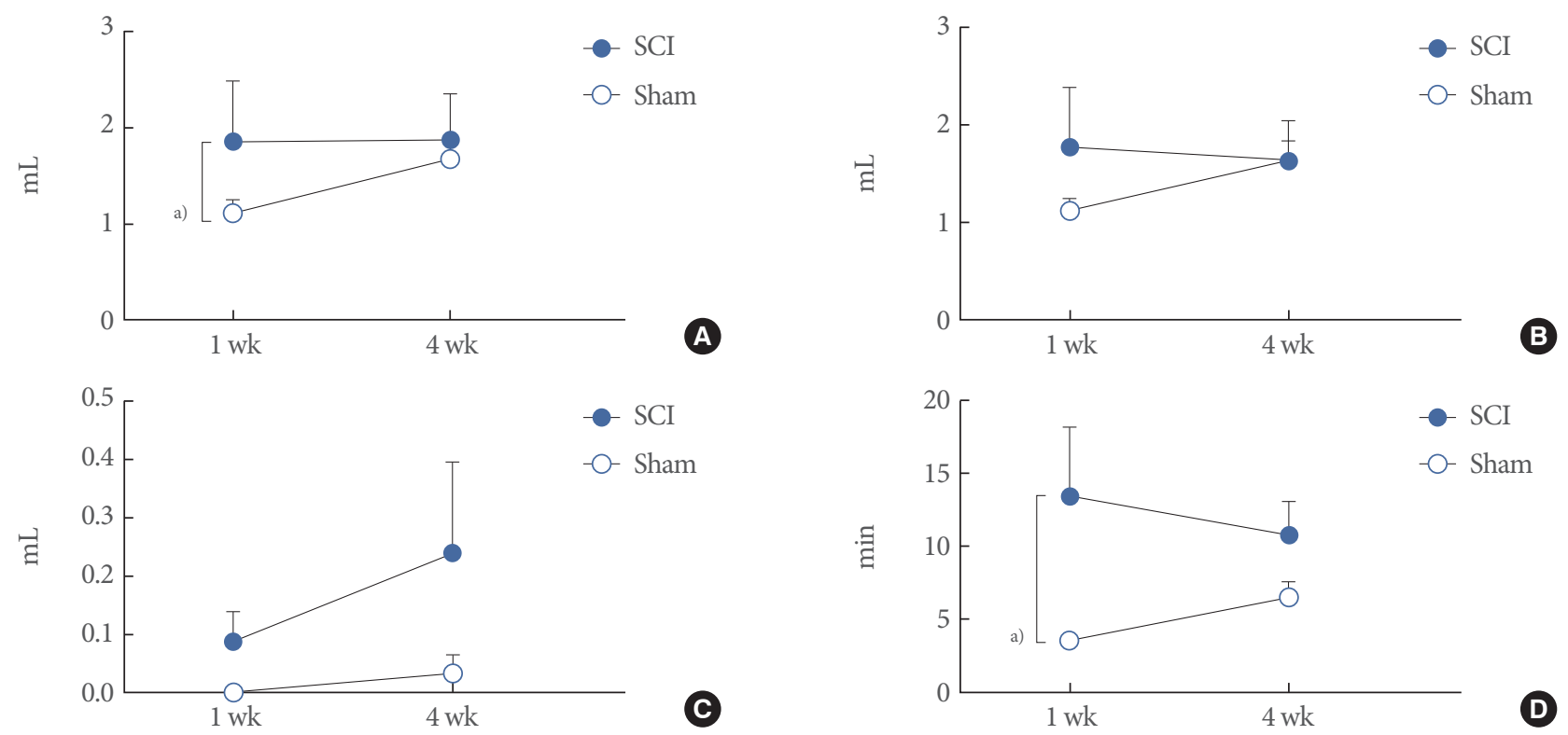

Fig. 4. The cystometric volume-related parameters of awake spinal cord injury (SCI) and Sham rats. (A) Bladder capacity. (B) Micturition volume. (C) Residual volume. (D) Micturition interval. Results are expressed as the mean \pm standard error of the mean. ${ }^{\text {a) }} \mathrm{P}<$ 0.05 (unpaired Student's t-test).

rats $(6.46 \pm 1.07$ minute) compared with sham rats 1 week after the injury ( $3.51 \pm 0.40$ minute; $\mathrm{P}<0.05$ ), although there were no significant differences at 4 weeks after the injury (Fig. 4).

\section{Parameters for Investigating Detrusor Overactivity During the Filling Phase}

All the SCI rats showed DO, but the sham rats did not. The DO frequency of the SCI rats 1 week after the injury $(2.06 \pm 0.41$ $\min ^{-1}$ ) decreased significantly compared with that at 4 weeks $(0.92 \pm 0.18$ minute; $\mathrm{P}<0.05)$. The DO pressure showed no significant difference between the SCI rats at $1(11.55 \pm 2.85 \mathrm{~cm}$ $\left.\mathrm{H}_{2} \mathrm{O}\right)$ and $4\left(8.40 \pm 1.87 \mathrm{cmH}_{2} \mathrm{O} ; \mathrm{P}>0.05\right)$ weeks after the injury, but showed a tendency for decreased pressure over the time course (Fig. 5).

\section{DISCUSSION}

The annual rate of SCI is approximately 12,000 new cases at risk in the United States. The average age at injury is 32.6 years, and even children comprise some percentages in these patients [12, 13]. Because this disease causes disablility throughout their lives, people with an SCI will continue to need medical care and various forms of personal assistance, especially for altered lower urinary tract function, which can lead to significant morbidity 

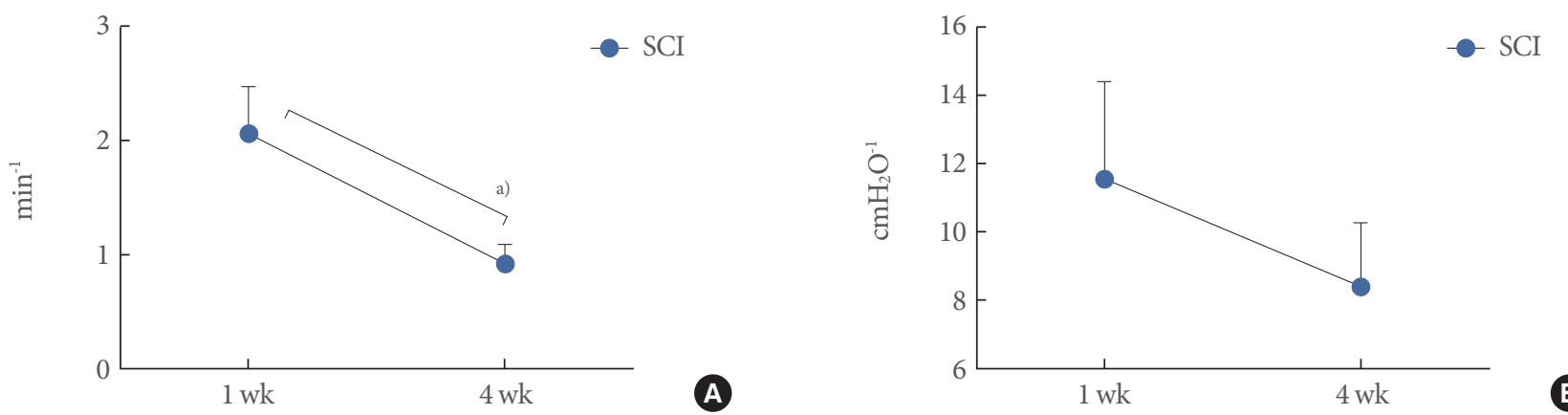

Fig. 5. Cystometric detrusor overactivity (DO)-related parameters during the filling phase in awake spinal cord injury (SCI) rats at 1 and 4 weeks after the injury. (A) DO frequency. (B) DO pressure. Results are expressed as the mean \pm standard error of the mean. ${ }^{\text {a) }} \mathrm{P}$ $<0.05$ (unpaired Student's t-test).

and mortality $[9,14,15]$. There is still much debate on urologic practice regarding follow-up and surveillance among spinal injury units, because of a lack of evidence on the long-term results of the lower urinary tract with SCI $[16,17]$.

Our principal questions in this study were how the DO would change over the time course of 1 month, and whether this would reflect the long-term changes in the bladder itself. To address these questions objectively we evaluated bladder function in SCI rats, by urodynamic testing with the simultaneous registration of IVP and IAP. In this study, the urodynamic findings in the SCI rats at 1 and 4 weeks after injury compared with the control rats in the same period showed decreased DO frequency without a change in DO pressure at 1 month after the injury compared with 1 week after the injury.

SCI rostral to the lumbosacral level causes lower urinary tract dysfunction, including detrusor hyperreflexia (DH) and detrusor-sphincter dyssynergia (DSD) [1-3]. Many authors have suggested that the long-term changes in the bladder are caused by the DSD $[9,18]$, which is defined as the presence of an involuntary contraction of the external sphincter during an involuntary detrusor contraction [8]. It causes high bladder pressure and increased residual urine, caused by the BOO, leading to urinary retention, higher intravesical pressure and structural bladder damage. Our study showed increased TP, increased MP, and an increased tendency for RV in rats 1 month after the injury, similar to the observation in the above studies.

Although the clinical significance of DSD is well established [19-21], we suggest the changes in the DO characteristics of the bladder itself as the causes of the long-term changes in lower urinary tract function. DH is the condition of uninhibited bladder contractions during the storage phase, which is known to be caused by a loss of the neural suppression to the micturition reflex center and the sustained activation of C-fibers after injury to the spinal cord [22]. In our study, the DO frequency decreased 1 month after the injury, compared with 1 week. The natural history of the lesions in SCI is usually divided into early, intermediate and late stages [23]. Immediately following the injury, complete disruption of nerve fibers and traumatic demyelination is observed within a few hours after injury [24]. The cellular and tissue changes in the spinal cord continue until the end stage is reached [23], which seems to be related to our result of decreased DO frequency, although there is no detailed information concerning plastic changes that may occur below the level of injury as a result of altered afferent inputs, reflex changes and transmitter neurochemistry.

Although the degree of DSD is associated with the severity of SCI, the function of the lower urinary tract is known to be recovered in part from bladder areflexia with time after incomplete SCI, which is demonstrated as the re-emergence of involuntary reflex micturition and detrusor hyperreactivity [4-6]. According to our data, the bladder 1 week after the injury already seemed to have recovered bladder reflex, according to the results of the DO during the filling phase and definite voiding phase. However, there seem to have been a period of areflexic bladder, because the rats showed increased BC and MI at 1 week after the injury. However, this increase disappeared 3 weeks later, which suggests the effect of hyperreflexic bladder.

In the present study, our urodynamic study with simultaneous registrations of IVP and IAP suggested that the long-term functional deficits of the bladder resulting from SCI are related to the effect of the DO characteristics as well as the DSD. The damage to the spinal cord can alter the balance of neuronal control over bladder activity, resulting in $\mathrm{DO}$, and the recovery process of the injured spinal cord can elicit changes in DO char- 
acteristics. The neurological basis of these late-phase changes remains poorly understood, but may provide important prognostic information about the long-term urological management in SCI patients.

\section{CONFLICT OF INTEREST}

No potential conflict of interest relevant to this article was reported.

\section{ACKNOWLEDGEMENTS}

This work was supported by a Inha University research grant.

\section{REFERENCES}

1. Yoshiyama M, Nezu FM, Yokoyama O, Chancellor MB, de Groat WC. Influence of glutamate receptor antagonists on micturition in rats with spinal cord injury. Exp Neurol 1999;159:250-7.

2. de Sèze M, Wiart L, de Sèze MP, Soyeur L, Dosque JP, Blajezewski S, et al. Intravesical capsaicin versus resiniferatoxin for the treatment of detrusor hyperreflexia in spinal cord injured patients: a doubleblind, randomized, controlled study. J Urol 2004;171:251-5.

3. Elliott S, Krassioukov A. Malignant autonomic dysreflexia in spinal cord injured men. Spinal Cord 2006;44:386-92.

4. de Groat WC, Kawatani M, Hisamitsu T, Cheng CL, Ma CP, Thor K, et al. Mechanisms underlying the recovery of urinary bladder function following spinal cord injury. J Auton Nerv Syst 1990;30 Suppl: S71-7.

5. Jonas U, Jones LW, Tanagho EA. Recovery of bladder function after spinal cord transection. J Urol 1975;113:626-8.

6. de Groat WC. Mechanisms underlying the recovery of lower urinary tract function following spinal cord injury. Paraplegia 1995;33: 493-505.

7. Weld KJ, Graney MJ, Dmochowski RR. Clinical significance of detrusor sphincter dyssynergia type in patients with post-traumatic spinal cord injury. Urology 2000;56:565-8.

8. Watanabe T, Rivas DA, Chancellor MB. Urodynamics of spinal cord injury. Urol Clin North Am 1996;23:459-73.

9. Shingleton WB, Bodner DR. The development of urologic complications in relationship to bladder pressure in spinal cord injured patients. J Am Paraplegia Soc 1993;16:14-7.
10. Lee T, Andersson KE, Streng T, Hedlund P. Simultaneous registration of intraabdominal and intravesical pressures during cystometry in conscious rats--effects of bladder outlet obstruction and intravesical PGE2. Neurourol Urodyn 2008;27:88-95.

11. Jin LH, Andersson KE, Kwon YH, Park CS, Yoon SM, Lee T. Substantial detrusor overactivity in conscious spontaneously hypertensive rats with hyperactive behaviour. Scand J Urol Nephrol 2009;43: 3-7.

12. Rabchevsky AG, Smith GM. Therapeutic interventions following mammalian spinal cord injury. Arch Neurol 2001;58:721-6.

13. DeVivo MJ, Go BK, Jackson AB. Overview of the national spinal cord injury statistical center database. J Spinal Cord Med 2002;25: 335-8.

14. Soden RJ, Walsh J, Middleton JW, Craven ML, Rutkowski SB, Yeo JD. Causes of death after spinal cord injury. Spinal Cord 2000;38: 604-10.

15. Garshick E, Kelley A, Cohen SA, Garrison A, Tun CG, Gagnon D, et al. A prospective assessment of mortality in chronic spinal cord injury. Spinal Cord 2005;43:408-16.

16. Pannek J, Göcking K, Bersch U. Long-term effects of repeated intradetrusor botulinum neurotoxin A injections on detrusor function in patients with neurogenic bladder dysfunction. BJU Int 2009; 104:1246-50.

17. Kuo HC. Clinical outcome and quality of life after enterocystoplasty for contracted bladders. Urol Int 1997;58:160-5.

18. McDonald JW, Sadowsky C. Spinal-cord injury. Lancet 2002;359: 417-25.

19. Blaivas JG, Sinha HP, Zayed AA, Labib KB. Detrusor-external sphincter dyssynergia. J Urol 1981;125:542-4.

20. Blaivas JG, Sinha HP, Zayed AA, Labib KB. Detrusor-external sphincter dyssynergia: a detailed electromyographic study. J Urol 1981;125:545-8.

21. Yalla SV, Blunt KJ, Fam BA, Constantinople NL, Gittes RF. Detrusor-urethral sphincter dyssynergia. J Urol 1977;118:1026-9.

22. Häbler HJ, Jänig W, Koltzenburg M. Activation of unmyelinated afferent fibres by mechanical stimuli and inflammation of the urinary bladder in the cat. J Physiol 1990;425:545-62.

23. Kakulas BA. The applied neuropathology of human spinal cord injury. Spinal Cord 1999;37:79-88.

24. Feigin I, Cravioto H. A histochemical study of myelin. A difference in the solubility of the glycolipid components in the central and peripheral nervous systems. J Neuropathol Exp Neurol 1961;20:245-54. 